\title{
Effect of added salt on preformed surface nanobubbles: A scaling estimate
}

\author{
Siddhartha Das \\ Physics of Fluids Group, Faculty of Science of Technology, University of Twente, P.O. Box 217, NL-7500 AE Enschede, The Netherlands
}

(Received 17 June 2011; revised manuscript received 11 August 2011; published 7 September 2011)

\begin{abstract}
In this paper we propose a scaling argument to quantify the role of added electrolyte salt in affecting the stability and the morphology of preformed surface nanobubbles on hydrophobic substrates like the water-OTS-silicon or the water-HOPG interfaces. The added salt controls the electric double layer formation as well as affects the zeta $(\zeta)$ potential at the air-water and solid-water interfaces. The resulting electrostatic wetting tension acts in conjunction with the air-water surface tension (analogous to electrowetting scenarios), thereby affecting the nanobubble morphologies. Weak $\zeta$ potential of the water-HOPG interface or the water-OTS-silicon interface at acidic $\mathrm{pH}$ ensures that the added salt will have imperceptible effect on the corresponding preformed surface nanobubbles, validating the experimental observations. However, at alkaline buffer $\mathrm{pH}$ for the OTS-silicon substrate, under certain system conditions, salt-induced $\zeta$ potential can be substantially high so that the properties of preformed surface nanobubbles will be affected. This paper will thus readdress the long-held universal notion that added salt, no matter in what concentration, will not influence the properties of preformed surface nanobubbles.
\end{abstract}

DOI: 10.1103/PhysRevE.84.036303

PACS number(s): 47.55.db, 68.08.-p, 68.03.-g

\section{INTRODUCTION}

Substrates in contact with water lead to formation of spherical caplike nanoscopic air-filled bubbles, popularly known as surface nanobubbles. These bubbles, most abundant for hydrophobic substrates, have received tremendous attention over the last decade with studies attempting to unravel the mystery behind their formation and quantify their properties as a function of different system parameters [1-38], most of which are well documented in a few recent review articles [39-41].

Considering the extremely high Laplace pressure inside the surface nanobubbles, owing to their radii of curvature of the order $100 \mathrm{~nm}$, the surface nanobubbles must disappear almost instantly after their formation. Quite inexplicably, however, surface nanobubbles are found to be extremely stable, and when left undisturbed, they remain in unchanged morphologies for several hours [16,22]. Changes in morphologies of surface nanobubbles, though, can be induced when system parameters are changed, e.g., when temperature is increased or solvent is changed [7,9,16,33,42]. Similar inferences, however, cannot be made for the added electrolyte salt. Experiments show that added electrolyte salt, no matter in what concentration, has no effect on the morphologies of preformed surface nanobubbles on HOPG substrate $[1,43]$. Similar observations have been made for surface nanobubbles on OTS-silicon-water interfaces for acidic buffer $\mathrm{pH}$ [43]. From these studies a general inference has been drawn that preformed surface nanobubbles will never be affected by addition of salt $[1,43]$, despite the fact that there has been no concrete theoretical justification supporting this inference.

In this paper, a theory is developed to elucidate the effect of added electrolyte salt on the morphologies of preformed surface nanobubbles. Both the water-substrate interface as well as the air-water interface (of the nanobubbles) are assumed to be charged [44-48] with a finite electric double layer (EDL) (characterized by the Debye-Huckel thickness $\lambda_{D}$ ), with the added salt dictating $\zeta$ potential [49] as well as the formation of the EDL at both these interfaces. The resulting electrostatic wetting tension that the nanobubble air-water interface experiences [44-48] acts in tandem with the air-water surface tension, and following the analogy of electrowetting scenarios [50] will potentially affect the nanobubble morphologies. With a model based on the calculation of electrostatic wetting tension for charged drops on charged substrates [44-48], scaling estimates are derived for the ratio of the electrostatic wetting tension to the air-water surface tension. The greater this ratio, the stronger will be the influence of the added salt on the properties of preformed surface nanobubbles. Estimates are provided for both HOPG as well OTS-silicon substrates as functions of their $\zeta$ potential for very large ionic concentration (i.e., cases for which $\lambda_{D} \ll h_{b}$, where $h_{b}$ is the characteristic length scale of nanobubbles) and very low ionic concentration (i.e., cases for which $\lambda_{D} \gg h_{b}$ ). Intrinsically low, $\mathrm{pH}$-independent $\zeta$ potential of the HOPG substrate results in negligible effect of the added salt on the preformed surface nanobubbles, confirming the experimental observations of Refs. [1,43]. Also for an OTS-silicon interface, where the substrate $\zeta$ potential depends on buffer $\mathrm{pH}$ and concentration of added salt [51-53], at acidic $\mathrm{pH}, \zeta$ potential remains small enough so that the effect of added salt is negligible (as observed by Ref. [43]). However, for alkaline buffer $\mathrm{pH}$ and high concentration of added salt (for such parameters there are no experiments), the $\zeta$ potential is shown to be substantially high, ensuring that added salt affects the properties (such as contact angle) of preformed surface nanobubbles on OTS-silicon substrates. Thus the present theory, which is probably the first quantitative study on the effect of added salt on preformed surface nanobubbles, not only validates the experimental observations of inconsequentiality of added salt on preformed nanobubbles for water-HOPG or water-OTS-silicon (at acidic buffer $\mathrm{pH}$ ) interfaces, but, more importantly, also demonstrates that for optimal combinations of system parameters that added salt may potentially influence the morphologies of preformed surface nanobubbles. Hence, we readdress the general notion that added salt, no matter under what condition, has no effect on preformed surface nanobubbles.

Apart from the extremely large stability, the other mystery concerning the surface nanobubbles is their extremely small contact angles, as compared to the macroscopic values. At 


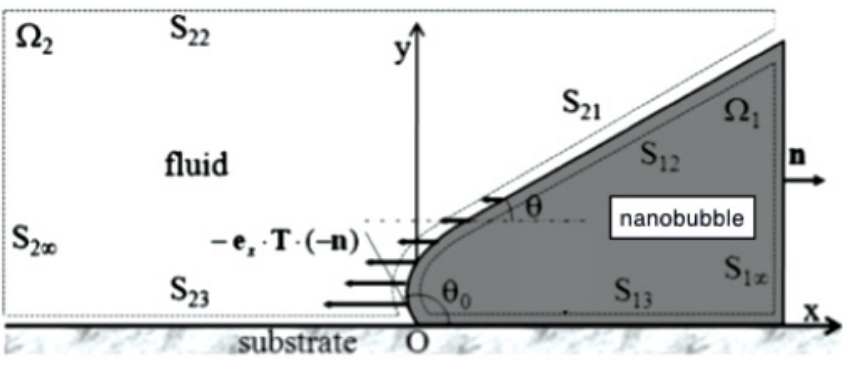

FIG. 1. Schematic of the nanobubble with the variation of the contact angle due to electrical effects. The microscopic angle $\theta_{0}$ is identical to the Young's angle (irrespective of the strength of the electric field) and $\theta$ is the electrical-effect-induced altered contact angle (macroscopic). This figure is similar to that of Ref. [48], with the charged drop being replaced by a surface nanobubble. In this figure the volumes of the different phases $\Omega_{i}$ and the bounding planes between these different phases $S_{i j}$ are also depicted.

the end of the paper we also discuss on how the scope (and limitation) of the present theory depends on the mechanism that is responsible for such an anomalously small nanobubble contact angle.

\section{THEORY}

\section{A. General model for electrostatic wetting tension}

Electrostatic wetting tension modifies the contact angle of a drop or bubble resting on a substrate from a microscopic value $\theta_{0}$ (identical to Young's angle, irrespective of the strength of the wetting tension [54]) to a different value $\theta$ (Fig. 1), such that

$$
\cos \theta=\cos \theta_{0}+\frac{W_{\mathrm{el}}}{\gamma},
$$

where $\gamma$ is the air-water surface tension and $W_{\mathrm{el}}$ is the electrostatic wetting tension (force per unit width).

The best-known example where Eq. (1) is encountered is electrowetting, where an externally applied voltage (of strength $V$ ) induces a wetting tension $W_{\mathrm{el}}=\epsilon_{0} \epsilon_{r} V^{2} / d$ [50] on a drop sitting on a dielectric (of thickness $d$ and relative permittivity $\epsilon_{r}$ ) effecting a change in the drop shape (i.e., a change in its macroscopic contact angle). For the present case, the electrostatic wetting tension is induced by the finite $\zeta$ potentials at the air-water and the liquid-solid interfaces (i.e., the role of the external voltage in electrowetting is now played by the substantially high $\zeta$ potentials), regulated by the EDL thickness (or concentration of added salt). We next quantify the electrostatic wetting tension experienced by a surface nanobubble; we follow the approach of Refs. [44-48], with the charged liquid drop of Refs. [44-48] being replaced by a surface nanobubble (Fig. 1).

For deriving the electrostatic wetting tension for the nanobubble we refer to Fig. 1. In Fig. 1, there are three distinct phases: the air-filled nanobubble (denoted as phase 1), the surrounding water (denoted as phase 2), and the solid substrate (denoted as phase 3 ). Also the bounding planes between any two phases $i$ and $j$ are denoted as $S_{i j}$. Further the volumes inside the nanobubble and the surrounding liquid are denoted as $\Omega_{1}$ and $\Omega_{2}$ respectively. Under these conditions, the electrostatic wetting tension at the air-water interface of the nanobubble is expressed as [44-48]

$$
W_{\mathrm{el}}=-\mathbf{e}_{x} \cdot \int_{S_{12}+S_{21}} \mathbf{T} \cdot(-\mathbf{n}) d S,
$$

where $\mathbf{T}$ is the electrostatic stress tensor given by

$$
\mathbf{T}=-\Pi \mathbf{I}+\epsilon_{0} \epsilon_{w}\left(\mathbf{E E}-\frac{1}{2} E^{2} \mathbf{I}\right) .
$$

In Eq. (2), $\Pi$ is the osmotic pressure, expressed through Poisson's equation as

$$
\nabla \Pi=\epsilon_{0} \epsilon_{w}(\nabla \cdot \mathbf{E}) \mathbf{E},
$$

and the rest of the terms are the Maxwell's stress. Also in the above equations $\mathbf{E}$ is the electric field vector (with $E$ being its magnitude), $\mathbf{n}$ is the outward unit normal vector to the surfaces, and $\mathbf{e}_{x}$ is the unit vector along positive $x$ direction (Fig. 1).

For a volume $\Omega$ enclosed by a surface $\Sigma$ (with $\Sigma$ lying entirely within a single phase), one may write for the electrostatic stress tensor (see Appendix A for derivation)

$$
\int_{\Sigma} \mathbf{T} \cdot \mathbf{n} d S=0 .
$$

Using Eq. (5) in Eq. (2), along with the definition of the closed surface $\Sigma_{2}$ (in the liquid domain), i.e., $\Sigma_{2}=S_{21}+$ $S_{22}+S_{23}+S_{2 \infty}$ (Fig. 1), one may write [under the condition that there is no electric field inside the nanobubble, which leads to $\left.\mathbf{e}_{x} \cdot \int_{S_{12}} \mathbf{T} \cdot(-\mathbf{n}) d S=0\right]$

$$
W_{\mathrm{el}}=\mathbf{e}_{x} \cdot\left[\int_{S_{22}+S_{23}+S_{2 \infty}} \mathbf{T} \cdot(-\mathbf{n}) d S\right] .
$$

Considering the correct representation of the unit vectors n for the individual planes $S_{22}, S_{23}$, and $S_{2 \infty}$, as well as the expression for the stress tensor $\mathbf{T}$ and the fact that $(\mathbf{E E}) \cdot \mathbf{n}=$ (n · E) E [44], one may simplify Eq. (6) as

$$
\begin{aligned}
W_{\mathrm{el}}= & {\left[\int_{S_{2 \infty}}\left(\Pi+\frac{\epsilon_{0} \epsilon_{w} E^{2}}{2}\right) d S\right] } \\
& -\int_{S_{22}+S_{23}}\left[\epsilon_{0} \epsilon_{w}(\mathbf{n} \cdot \mathbf{E}) \mathbf{E}\right] \cdot \mathbf{E}_{x} d S
\end{aligned}
$$

Note the second term on RHS of Eq. (7) will contribute only when the electric fields $\mathbf{E}_{S_{23}}$ and $\mathbf{E}_{S_{22}}$ also have a component along the axial direction, i.e., $\mathbf{E}_{S_{23}} \cdot \mathbf{e}_{x} \neq 0$ and $\mathbf{E}_{S_{22}} \cdot \mathbf{e}_{x} \neq 0$.

\section{B. Scaling estimates}

Exact estimation of the different terms in Eq. (7) is beyond the scope of the present continuum analysis. First, the air-water interface potential is not known a priori. As has been shown in several recent studies, this potential is a function of orientation and structuring of the dipolar water molecules [55-58] as well as the spatial distribution of the ions (from the added salt) at the air-water interface [59-61], and extensive molecular simulations are needed to quantify it. The second difficultly is the specification of the ionic distribution (or to be more precise the EDL) around the region where the contact angle changes from the microscopic (Young's value) to macroscopic value. EDL thickness being typically of the order of few nanometers, it is of similar length scale over which this change in the contact 
angle occurs, thereby making the exact specification of EDL ion distribution at this transition region extremely difficult, and it must be obtained by suitable molecular simulation experiments.

On the other hand, the possible impact of the added salt on the nanobubble morphologies can be obtained by a scaling estimate of the electrostatic wetting tension, and using that estimate to obtain the ratio of the wetting tension to the surface tension, which dictates the resulting alteration of the contact angle [see Eq. (1)]. In the following subsections we shall discuss this scaling and the resulting $W_{\mathrm{el}} / \gamma$ ratio for two extreme cases, namely, that for very large ionic concentration (i.e., when $\lambda_{D} \ll h_{b}$ ) and very small ionic concentration (i.e., when $\lambda_{D} \gg h_{b}$ ), under the condition that $\zeta$ potential at the water-solid and water-air interfaces are $\zeta_{1}$ and $\zeta_{2}$, respectively (with $\left|\zeta_{2}\right| \ll\left|\zeta_{1}\right|$ ).

\section{Case with $\lambda_{D} \ll h_{b}$}

For this case, the magnitude of the electric field at locations where only the electric field induced by the EDL at the solidwater interface contributes scales as

$$
E \sim \frac{\zeta_{1}}{\lambda_{D}}
$$

Similarly, the electric field at locations where only the electric field induced by the EDL at the air-water interface contributes scales as

$$
E \sim \frac{\zeta_{2}}{\lambda_{D}}
$$

Note that here the length scale for determining the electric field is the EDL thickness $\lambda_{D}$, because outside the EDL (which is much smaller than the nanobubble height $h_{b}$ ) the electric field is negligible. For the same reason $\int_{S_{2 \infty}}$ implies integration over a distance $\lambda_{D}$. Also along $S_{2 \infty}, E=\zeta_{1} / \lambda_{D}$. Further, under these conditions the osmotic pressure $\Pi$ scales as [we need $\Pi$ along $S_{2 \infty}$, see Eq. (7), so that the corresponding $\left.E \sim \zeta_{1} / \lambda_{D}\right]$

$$
\Pi \sim \epsilon_{0} \epsilon_{w} E^{2} \sim \epsilon_{0} \epsilon_{w} \frac{\zeta_{1}^{2}}{\lambda_{D}^{2}} .
$$

Consequently,

$$
\int_{S_{2 \infty}}\left(\Pi+\frac{\epsilon_{0} \epsilon_{w} E^{2}}{2}\right) d S \sim \epsilon_{0} \epsilon_{w} \frac{\zeta_{1}^{2}}{\lambda_{D}} .
$$

Also, the plane $S_{22}$ is outside the EDL of either of the interfaces, $E_{S_{22}}=0$, so that

$$
\int_{S_{22}}\left[\epsilon_{0} \epsilon_{w}(\mathbf{n} \cdot \mathbf{E}) \mathbf{E}\right] \cdot \mathbf{E}_{x} d S=0 .
$$

Calculation of $\int_{S_{23}}\left[\epsilon_{0} \epsilon_{w}(\mathbf{n} \cdot \mathbf{E}) \mathbf{E}\right] \cdot \mathbf{E}_{x} d S$ is nontrivial. This is because it has a finite value close to the TPCL (in the region where the EDL from the nanobubble-water interface interacts with the EDL at the solid substrate); however, at locations of the plane $S_{22}$ where the EDL from the nanobubble-water interface ceases to exist, it vanishes because at such locations the electric field is normal to the surface, i.e., $\mathbf{E} \cdot \mathbf{e}_{x}=0$. Hence one can write

$$
\int_{S_{23}}\left[\epsilon_{0} \epsilon_{w}(\mathbf{n} \cdot \mathbf{E}) \mathbf{E}\right] \cdot \mathbf{E}_{x} d S \sim \epsilon_{0} \epsilon_{w} \frac{\varphi_{\mathrm{TPCL}}^{2}}{\lambda_{D}},
$$

where $\varphi_{\mathrm{TPCL}}$ is the potential at the TPCL.
Following the derivation of Kang et al. [45], one can obtain $\varphi_{\text {TPCL }}$ and that can be used in Eq. (13), so as to obtain

$$
\int_{S_{23}}\left[\epsilon_{0} \epsilon_{w}(\mathbf{n} \cdot \mathbf{E}) \mathbf{E}\right] \cdot \mathbf{E}_{x} d S \sim \epsilon_{0} \epsilon_{w} \frac{\zeta_{1} \zeta_{2}}{\lambda_{D}} .
$$

As $\left|\zeta_{2}\right| \ll\left|\zeta_{1}\right|, \frac{\zeta_{1} \zeta_{2}}{\lambda_{D}} \ll \frac{\zeta_{1}^{2}}{\lambda_{D}}$. Consequently, using Eqs. (11), (12), and (14) in Eq. (7), one may write (for $\lambda_{D} \ll h_{b}$ )

$$
W_{\mathrm{el}} \sim \epsilon_{0} \epsilon_{w} \frac{\zeta_{1}^{2}}{\lambda_{D}} .
$$

\section{Case with $\lambda_{D} \gg h_{b}$}

For this case the equations equivalent to Eq. (8) and Eq. (9) are

$$
\begin{aligned}
& E \sim \frac{\zeta_{1}}{h_{b}}, \\
& E \sim \frac{\zeta_{2}}{h_{b}} .
\end{aligned}
$$

The important difference that can be witnessed between Eqs. (8) and (9) and the above equations is that the length scale for determining the electric field is now the nanobubble dimension (i.e., its height) (this is so because the EDL electric field at locations substantially distant from the nanobubble does not contribute to the wetting tension experienced by the nanobubble).

Before proceeding further, we now state an important assumption that will be used in the following derivation. The nanobubble dimension being significantly smaller than the EDL thickness, we assume that at any point surrounding the nanobubble, the electric field of the EDL from a particular interface (i.e., either the solid-water or nanobubble-water interface) is not diminished by the corresponding EDL screening effect [49] and is always either $\zeta_{1} / h_{b}$ or $\zeta_{2} / h_{b}$. One can justify such an assumption by noting that the screening effect will lead to decay of the electrostatic potential that scales as $\exp \left(-h_{b} / \lambda_{D}\right) \sim 1-h_{b} / \lambda_{D} \sim 1$ (as $\left.\lambda_{D} \gg h_{b}\right)$. The usefulness of such an assumption is that at any point around the nanobubble, one can ignore the intricate EDL dynamics resulting from the interactions of the EDLs created at the two interfaces (water-substrate and air-water interfaces) and consider the electric field to be simply the linear superposition of the electric fields induced at that point by these two different charged interfaces with the surrounding medium being that of deionized water.

Based on this idea, we provide the scalings of the electric fields at the different planes $\left(S_{22}, S_{2 \infty}, S_{23}\right)$.

For all of the planes, the electric field along $y$ is contributed by the fields of both the interfaces, whereas the electric field along $x$ is contributed by the field of only the air-water interface.

Hence, one can write for any plane $S_{2 j}$ (where $j=2,3, \infty$ )

$$
\mathbf{E}_{S_{2 j}} \sim\left(\left|\mathbf{E}_{2 j}^{\mathrm{aw}}\right|\right) \mathbf{e}_{x}+\left(\left|\mathbf{E}_{2 j}^{\mathrm{aw}}\right|+\left|\mathbf{E}_{2 j}^{\mathrm{sw}}\right|\right) \mathbf{e}_{y},
$$

where $\left|\mathbf{E}_{2 j}^{\mathrm{aw}}\right| \sim \zeta_{2} / h_{b}$ and $\left|\mathbf{E}_{2 j}^{\mathrm{sw}}\right| \sim \zeta_{1} / h_{b}$ are the contributions of the electric fields from the air-water and solid-water interfaces, respectively. 
As $\left|\zeta_{2}\right| \ll\left|\zeta_{1}\right|$, one can write

$$
\mathbf{E}_{S_{2 j}} \sim \frac{\zeta_{2}}{h_{b}} \mathbf{e}_{x}+\frac{\zeta_{1}}{h_{b}} \mathbf{e}_{y} .
$$

To evaluate the electrostatic wetting tension, one first needs the magnitude of the electric field along $S_{2 \infty}$, which is [from Eq. (19)]

$$
E_{S_{2 \infty}} \sim \sqrt{\frac{\zeta_{2}^{2}}{h_{b}^{2}}+\frac{\zeta_{1}^{2}}{h_{b}^{2}}} \sim \frac{\zeta_{1}}{h_{b}} .
$$

Therefore the osmotic pressure along $S_{2 \infty}$ scales as

$$
\Pi \sim \epsilon_{0} \epsilon_{w} E_{S_{2 \infty}}^{2} \sim \epsilon_{0} \epsilon_{w} \frac{\zeta_{1}^{2}}{h_{b}^{2}} .
$$

Also, $\int_{S_{2 \infty}}$ implies integration over a distance $h_{b}$. Consequently, one can write

$$
\int_{S_{2 \infty}}\left(\Pi+\frac{\epsilon_{0} \epsilon_{w} E^{2}}{2}\right) d S \sim \epsilon_{0} \epsilon_{w} \frac{\zeta_{1}^{2}}{h_{b}} .
$$

For either of the planes $S_{22}$ and $S_{23}$, one can write [using Eq. (19)]

$$
\begin{aligned}
\mathbf{n} \cdot \mathbf{E} & \sim \frac{\zeta_{1}}{h_{b}}, \\
\mathbf{E} \cdot \mathbf{e}_{x} & \sim \frac{\zeta_{2}}{h_{b}}, \\
{\left[\epsilon_{0} \epsilon_{w}(\mathbf{n} \cdot \mathbf{E}) \mathbf{E}\right] \cdot \mathbf{e}_{x} } & \sim \epsilon_{0} \epsilon_{w} \frac{\zeta_{1} \zeta_{2}}{h_{b}^{2}} .
\end{aligned}
$$

Further, neglecting the small distance over which the contact angle of the bubble changes from $\theta_{0}$ to $\theta$, one can relate the distance along $S_{23}$ (we call it $l_{S_{23}}$ ) to the distance along $S_{22}$ (we call it $l_{S_{22}}$ ) as

$$
l_{S_{22}}=l_{S_{23}}+h_{b} / \tan (\theta) \text {. }
$$

Also note that signs of $(\mathbf{E} \cdot \mathbf{n})_{S_{23}}$ and $(\mathbf{E} \cdot \mathbf{n})_{S_{22}}$ are opposite. Therefore using Eq. (25) and Eq. (26), one will get

$$
\int_{S_{22}+S_{23}}\left[\epsilon_{0} \epsilon_{w}(\mathbf{n} \cdot \mathbf{E}) \mathbf{E}\right] \cdot \mathbf{E}_{x} d S \sim \epsilon_{0} \epsilon_{w} \frac{\zeta_{1} \zeta_{2}}{h_{b} \tan \theta}
$$

Except for very small $\theta$, one can always write $\frac{\zeta_{1} \zeta_{2}}{h_{b} \tan \theta}<\frac{\zeta_{1}^{2}}{h_{b}}$, so that from Eq. (7), one can write that for $\lambda_{D} \gg h_{b}$,

$$
W_{\mathrm{el}} \sim \epsilon_{0} \epsilon_{w} \frac{\zeta_{1}^{2}}{h_{b}} .
$$

Note that the estimates of wetting tension for the two limiting cases [Eqs. (15)-(28)] bear close resemblance with the classical expression of electrowetting (on dielectric) induced wetting tension $\left(W_{\mathrm{el}}=\frac{\epsilon_{0} \epsilon_{r} V^{2}}{d}\right)$, with the length $d$ being replaced by the length scale of the problem $\left(\lambda_{D}\right.$ or $h_{b}$, depending on the relative EDL thickness), the external voltage $V$ being replaced by the solid-water substrate $\zeta$ potential $\left(\zeta_{1}\right)$, and the relative permittivity of the dielectric $\epsilon_{r}$ being replaced by the relative permittivity of water $\epsilon_{w}$.

\section{EFFECT ON NANOBUBBLE PARAMETERS}

To understand the consequence of the added electrolyte salt on the morphologies of preformed surface nanobubbles, we will compute the dimensionless number $R$, defined as

$$
R=\frac{W_{\mathrm{el}}}{\gamma},
$$

expressing the ratio of the electrostatic wetting tension to the air-water surface tension. From Eq. (1), it can be clearly seen that the alteration of nanobubble contact angle due to electrostatic effects is governed by this ratio $R$. In this section, $R$ is estimated for both HOPG and OTS-silicon substrates and used to obtain the possible consequences of the added electrolyte in lowering the nanobubble contact angles [using Eq. (1)].

\section{A. Zeta potential and ratio $\boldsymbol{R}$ for HOPG substrate}

Variation of $R$ for the HOPG substrate for the extreme cases of very large and very small ionic concentration is shown in Fig. 2. The $\zeta$ potential of HOPG being intrinsically small (and virtually independent of the buffer $\mathrm{pH}$ or the ionic concentration), $R$ is substantially small for either of the ranges of ionic concentration. Consequently, as has been demonstrated in the experiments $[1,43]$, the added electrolyte salt (no matter in what concentration) will have no impact on the morphology of preformed surface nanobubbles. It is interesting to note that for weak ionic concentration (or the case for which $\lambda_{D} \gg h_{b}$ ), $R$ is independent of the ionic concentration as it is primarily governed by the ionic concentration-independent $\zeta$ potential of the solid-water interface and the nanobubble dimensions $h_{b}$ [see Eq. (28)].

\section{B. Zeta potential and ratio $\boldsymbol{R}$ for OTS-silicon substrate}

Hydrophilic bare silica substrate is converted to extremely hydrophobic (ideal for producing stable surface nanobubbles)

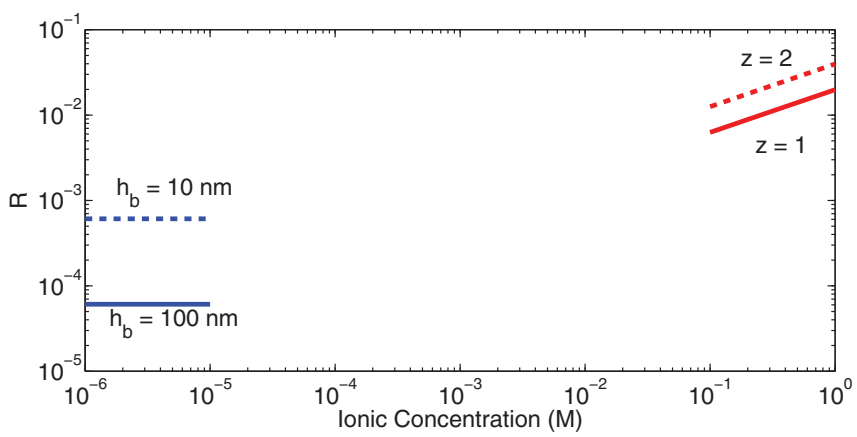

FIG. 2. (Color online) Variation of the ratio $R$ for HOPG for very large and very small concentration [the EDL thickness $\lambda_{D}$ is related to the ionic concentration (in moles/liter i.e., $c_{M}$ ) as $\lambda_{D}=\sqrt{\frac{\epsilon_{0} \epsilon_{w} k_{B} T}{2 \times 10^{3} c_{M} N_{A} e^{2} z^{2}}}$, where $N_{A}$ is the Avogadro number, $e$ is the electronic charge, and $z$ is the ionic valence]. In these plots we take a concentration independent $\zeta$ potential of $-25 \mathrm{mV}$ [66]. For very large concentration, (15) suggests a dependence of wetting tension on $\lambda_{D}$, and hence $R$ varies with ionic concentration and the valence $z$. However, for very small concentration, $R$ is independent of $\lambda_{D}$ [see (28)] and depends only on the nanobubble dimension $h_{b}$. 




FIG. 3. (Color online) Variation of $\zeta$ potential with buffer $\mathrm{pH}$ for bare silica nanochannels for both very large as well as for very small ionic concentration. Results from present simulation are compared to the corresponding experimental values [63-65] (the black circles correspond to $c_{M}=0.1 \mathrm{M}$, whereas the red diamonds correspond to $c_{M}=0.01 \mathrm{M}$ )]. The constant parameters used here are (following Ref. [53]) $\Gamma=5.0 \mathrm{~nm}^{-2}, C_{p, 1}=1.0 \mathrm{~F} / \mathrm{m}^{2}, C_{p, 2}=1.0 F / \mathrm{m}^{2}, K_{a 2}=$ $10^{-6.73}, K_{M}=10^{-0.25}, p \mathrm{H}_{\mathrm{pzc}}=3.0$, and $T=298 \mathrm{~K}$.

OTS-silicon substrate by providing molecular coating of OTS on the bare silica. Following Ref. [51], one can consider the wall charge density of the OTS-silicon as some fraction $f$ (this fraction depends on the extent of OTS treatment to the bare silica, and we always have $0<f<1$; greater extent of OTS coverage implies smaller $f$, hence for bare silica $f=1$ ) of the wall charge density of the bare silica $\sigma_{0}$. Therefore we first need to calculate $\sigma_{0}$ for bare wall silica. Bare wall charge density and the resulting $\zeta$ potential for the silica is determined by the chemical reaction between the silica wall and the ions in the system, and consequently is a function of the buffer $\mathrm{pH}$, the bulk ionic concentration, and the relative EDL thickness. There have been several studies that provide theoretical models to calculate the bare silica wall $\zeta$ potential as a function of the reactions of the substrate with the ions in the EDL $[52,53,62]$. In this paper we will follow the model proposed by Wang and Revil [53]. The important details of the model and how it has been employed in the present context to calculate the $\zeta$ potential for bare silica as well OTS-silicon (with a given $f$ ) substrates are summarized in Appendix B. First, we plot the $\mathrm{pH}$-dependent variation of $\zeta$ potential of the bare silica wall for small and large ionic concentrations (Fig. 3), and the results are found to match well with the experimental results [63-65] (Fig. 3).

In Fig. 4 we show the corresponding variation of the $\zeta$ potential for the OTS-silicon substrate for $f=0.9$. This $\zeta$ potential can be employed to obtain the wetting tension $W_{\mathrm{el}}$ [using Eq. (15) and Eq. (28)], the ratio $R$ [using Eq. (29)], and the lowering of the angle $\Delta \theta$ [using Eq. (1)] for surface nanobubbles on OTS-silicon substrates. Variation of $\Delta \theta$ is shown in Fig. 5, and one can clearly witness that under suitable conditions, added electrolyte salt can lead to notable variation of the contact angle of preformed surface nanobubbles on OTSsilicon-water interfaces.

\section{DISCUSSION}

Before discussing the results, we refocus our attention on the scaling estimates of Eq. (15) and Eq. (28), which clearly

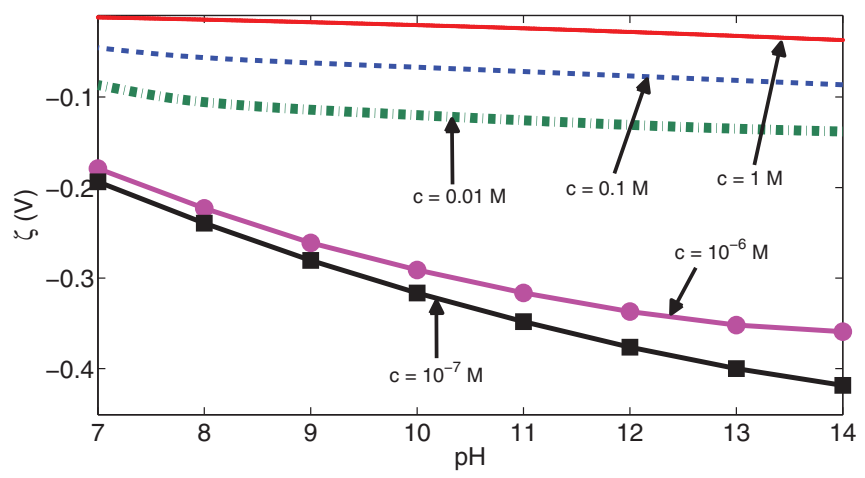

FIG. 4. (Color online) Variation of $\zeta$ potential with buffer $\mathrm{pH}$ for OTS-silicon substrates for both very large as well as very small ionic concentration for $f=0.9$. The constant parameters used here are identical to those used in Fig. 3.

show the governing influence of the relevant length scale for the problem (either the EDL thickness $\lambda_{D}$ or the nanobubble dimension $h_{b}$ ). When concentration of added salt is very high, the screening of the interface charge occurs over very small distances, leading to very small values of $\lambda_{D}\left(\ll h_{b}\right)$. This implies that the gap thickness of the hypothetical capacitor (i.e., the distance over which voltage drop is relevant), charged by the charges of the EDL, is $\lambda_{D}$. Physically such large crowding of the EDL ions may induce several nontrivial effects that are not considered in this basic EDL-based model for surface nanobubbles. One such factor may be the effect of finite capacitance of the Stern layer of the EDL, which deviates from the bulk capacitance value of $\epsilon_{0} \epsilon_{w} / \lambda_{D}$ [67]. The other nontrivial consequence of large ionic concentration is the finite ion size effect (known as the Steric effect), which may become important in the estimation of the electrostatic wetting tension [48]. However, as we are mostly interested in the scaling estimates (rather than the exact values) of wetting tension, we can safely ignore these effects. Despite the possibilities of such nontrivial electrochemical effects for

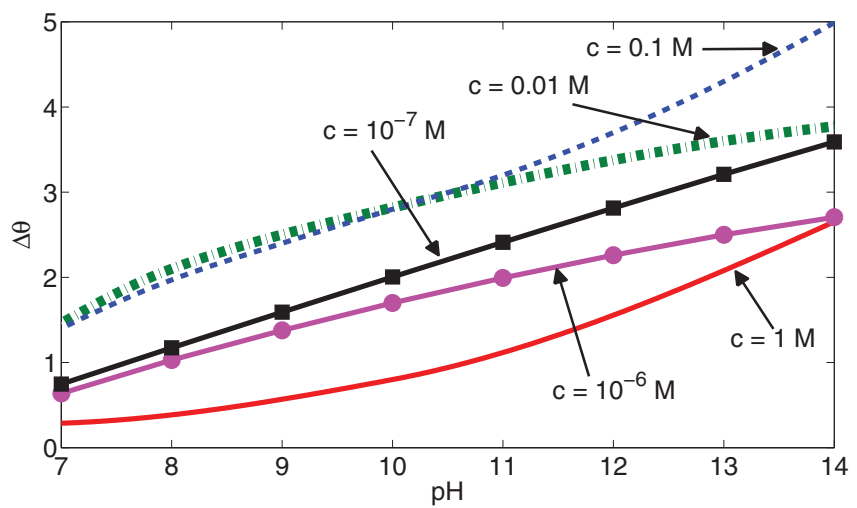

FIG. 5. (Color online) Variation of $\Delta \theta$ (where $\Delta \theta=\theta_{0}-\theta$ ) of preformed surface nanobubbles on OTS-silicon-water interface with buffer $\mathrm{pH}$ for both very large as well as very small ionic concentration (for the case with very small ionic concentration, we use $h_{b}=30 \mathrm{~nm}$ ) for $f=0.9$. In these plot we consider $\theta_{0}=\cos ^{-1}\left(\frac{\gamma_{\mathrm{sl}}-\gamma_{\mathrm{sv}}}{\gamma_{\mathrm{lv}}}\right)$, with $\gamma_{\mathrm{sl}}-\gamma_{\mathrm{sv}}=0.025 \mathrm{~N} / \mathrm{m}$ and $\gamma_{\mathrm{lv}}=0.072 \mathrm{~N} / \mathrm{m}$. 
the case with $\lambda_{D} \ll h_{b}$, the relevant choice of length scale for the scaling becomes straightforward and equal to the EDL thickness $\lambda_{D}$. Such a natural choice of the length scale is equally possible for the other case (i.e., $\lambda_{D} \gg h_{b}$ ) and is equal to $h_{b}$. However, the critical issue for this case $\left(\lambda_{D} \gg h_{b}\right)$ is the appropriate choice of the value of $h_{b}$. We choose $h_{b}$ as the smallest of the nanobubble dimension, which becomes equal to its height for acute contact angles. Nanobubble height depends on the experimental procedures and may show a wide range. However, values of around $h_{b} \sim 30 \mathrm{~nm}$ have been observed in a large number of experiments [2,4], including the study by Zheng et al. [43], whose experimental results of inconsequentiality of added salt on preformed surface nanobubbles have been validated by the present theory. Also such a value implies that we can safely use for very large ion concentration $\lambda_{D} \ll h_{b}$ as well as for very small ion concentration $\lambda_{D} \gg h_{b}$. Thus, we use this value to obtain the scaling estimate and hence lowering of the contact angle $\Delta \theta$ for the case with $\lambda_{D} \gg h_{b}$ (Fig. 5).

The intrinsically low $\zeta$ potential of the HOPG substrate ensures that the corresponding wetting tension invariably remains small enough to never affect the morphologies of the preformed surface nanobubbles (Fig. 2). This finding is crucial as it establishes the present theory by validating the well-known experimental results $[1,43]$. The case for the OTS-silicon substrate is much more nontrivial as it requires the exact calculation of the $\mathrm{pH}$ and ionic concentration-dependent $\zeta$ potential. In spite of the present study being primarily a scaling analysis, this explicit calculation of the $\zeta$ potential is important, as one can witness more than one order of variation of the $\zeta$ potential depending on the ionic concentration and the $\mathrm{pH}$ values (see Figs. 3 and 4). The $\mathrm{pH}$ and ionic concentration dependencies of the bare silica $\zeta$ potential matches well with the well-established experimental results. The $\zeta$ potential for OTS-silicon is always smaller than the corresponding values for the bare silica substrate. We only study the case for $f=0.9$, but for smaller values of $f$ (indicating greater coverage of OTS-silicon) even smaller values of the $\zeta$ potential of OTS-silicon substrate result. The central results of this paper are provided in Fig. 5. This figure clearly shows that for acidic (or even neutral $\mathrm{pH}$ ), irrespective of the concentration of the added salt, the changes in the morphologies of surface nanobubbles are very small, validating the observations of Ref. [43]. Thus through Figs. 2 and 5, we establish the experimental observations of inconsequentiality of added salt on preformed nanobubbles on HOPG or OTS-silicon (at acidic $\mathrm{pH}$ ) substrates. However, for suitable choices of the parameters [i.e., ideal combinations of ionic concentrations and $\mathrm{pH}$ (very alkaline)] the electrostatic wetting effect of the added salt can lower the nanobubble contact angle by 5 degrees. This is a substantially large variation that can be easily verified by the sophisticated experimental techniques that are being presently employed to study surface nanobubbles [39-41]. Remarkably, the increase in ionic concentration does not monotonically increase the effect of the added salt. This is because, though at larger ionic concentration $\lambda_{D}$ is small (thereby increasing $\left.W_{\mathrm{el}}\right)$, there is also a significant lowering of the wall $\zeta$ potential (see Figs. 3 and 4 and Refs. [63-65]), which will accordingly lower the $W_{\mathrm{el}}$ (as $W_{\mathrm{el}} \sim \zeta^{2}$ ). Thus only at optimum conditions of ionic concentration (large enough to ensure substantially small $\lambda_{D}$ and small enough to ensure substantially large $\zeta$ potential) can one witness the maximum effect of the added salt. Hence one witnesses the maximum effect for $c=0.1 \mathrm{M}$ or $c=0.01 \mathrm{M}$, whereas at the same time the effect for very small concentration $c=10^{-6} \mathrm{M}$ or $c=10^{-7} \mathrm{M}$ (for these scaling the relevant length scale is $h_{b}$ ) is more than that for very large concentration $c=1 \mathrm{M}$. This shows that the wetting tension effect is determined by the mutually counterbalancing influences induced by the addition of the salt, and an indiscriminate increase in the added salt may not always enhance the effect.

As an additional comment, it is worth mentioning that conditions of high alkalinity and weak OTS coverage (greater OTS coverage will substantially lower the wall $\zeta$ potential so that the effect of added salt will go down) are not always used by experimentalists studying surface nanobubbles. High alkalinity in the solution may cause damage to the AFM [43], whereas weak OTS coverage may lead to formation of only sparsely crowded surface nanobubbles. Thus, it may be argued that under the conditions that normally prevail for experiments involving surface nanobubbles, the effect of added salt may not be important in altering the morphologies of preformed surface nanobubbles. But, as we have demonstrated, under suitable conditions, added salt will have a significant impact on preformed surface nanobubbles, and this point must be well emphasized when discussing the effect of electrolyte salt on surface nanobubbles.

\section{A. Scope and limitation of the proposed theory}

The scope (and limitation) of the present theory depends, to a large extent, on the mechanism that lowers the nanobubble contact angle in comparison to the macroscopic contact angle by 30-50 degrees [35,41]. Ducker [35] hypothesized that one of the reasons may be the possible impurity-induced lowering of the liquid-vapor surface tension, which lowers the contact angle (by Young's law). In a couple of recent papers $[36,38]$, we provided definite quantitative arguments supporting this hypothesis. The effect of added salt will be even more pronounced in such a scenario, as shown below.

Without consideration of any impurities or no reduction in liquid-vapor surface tension [i.e., $\gamma_{\mathrm{lv}}=0.072 \mathrm{~N} / \mathrm{m}$ and contact angle equal to $70^{\circ}$ (i.e., macroscopic contact angle for water silica substrate)], we get [from Eqs. (15) and (28)], for $\zeta \sim-0.2 \mathrm{~V}$ and $\lambda_{D} \sim 10 \mathrm{~nm}$ (or $h_{b} \sim 10 \mathrm{~nm}$ ), $W_{\mathrm{el}} \sim 3 \times$ $10^{-3} \mathrm{~N} / \mathrm{m}$ and $\Delta \theta \sim 3^{\circ}$. Using identical parameters for the case where there are impurities (so that the reduced value of the surface tension $\gamma_{\mathrm{lv}}$ is approximately $0.03 \mathrm{~N} / \mathrm{m}$ and the contact angle $30^{\circ}$, i.e., closer to the experimental values $[35,41]$ ), we get from the present model $\Delta \theta \sim 11^{\circ}$.

Some very recent (unpublished) numerical simulations [68] have proposed an altogether different reason for the anomalously small contact angle of the surface nanobubbles. They suggest that the surface nanobubbles actually float on micropancakes [69-72], similar to the experimental observations of Zheng et al. [69]. Under such a condition the nanobubble never comes in contact with the solid substrate, and the gas layer below the nanobubble effectively renders the wall more hydrophobic, causing a substantial lowering of the 
contact angle. Brenner and Lohse [23], in their proposed theory for nanobubble stability, also argued the presence of such a dense layer of gas (though in dynamic equilibrium) at the nanobubble three-phase contact line, providing a continuous influx that prevents the nanobubbles from getting dissolved. It is rather nonintuitive to connect the present theory to such a hypothesis, as the present theory is based on the large charging of the solid substrate (in contact with water), with the surface nanobubble sitting on the solid substrate. However, in the case there are charged impurity molecules in the system, they can get adsorbed at these gaseous layers, making them charged (such charging of gaseous layers by adsorption of surfactant molecules is well known; see Ref. [73]), which induces electric double layers in the presence of added salt, so that the resulting electrostatic wetting tension can still affect the surface nanobubbles. However, for relatively clean experimental set ups it is likely that the impurities exist only in small concentration, which will lead to relatively weak charging of these interfaces so that the effect of added salt on surface nanobubbles, as proposed in the present theory, will get substantially reduced. It is important to note in this context that the gas enrichment at the nanobubble threephase contact line occurs due to the effects like van der Waals interactions between the gas and the solid walls that drive the gas molecules toward the nanobubble three-phase contact line [23,74]. Therefore, effects like van der Waals interactions strongly dictate the formation mechanism of the nanobubbles and hence will have substantial impact on the present theory of the salt effect on preformed surface nanobubbles.

Over the recent years, there has been considerable conjecture on the possible impact of line tension effect on nanoscale drops and bubbles [75], with the line tension effect scaling as inverse of the contact radius of the drops and bubbles. However, calculations clearly reveal that the line tension $T_{l}$ for such systems is typically $T_{l} \sim 10^{-11}-10^{-12} \mathrm{~N}[75,76]$, so that for a nanobubble with contact radius $r_{b} \sim 100 \mathrm{~nm}$, its influence, quantified by $T_{l} /\left(r_{b} \gamma_{\mathrm{lv}}\right) \sim 10^{-3}-10^{-4}$, is negligible. Thus the present theory will most likely be not affected by the line tension effect.

\section{v. CONCLUSIONS}

In this paper we analyze the effect of added salt in the morphologies of preformed surface nanobubbles by providing useful scaling estimates as functions of the system parameters. In addition to validating the experimental observations of inconsequentiality of added salt for nanobubbles on HOPG substrate or OTS-silicon substrate at acidic $\mathrm{pH}$, it is also demonstrated that in an alkaline medium for a suitably charged hydrophobic substrate, optimum concentration of added electrolyte salt can significantly alter the properties of preexisting surface nanobubbles. We thus attempt to readdress the idea that added salt under no condition can ever affect the properties of preformed surface nanobubbles [39,43]. We also discuss how the possible scope (and limitations) of this theory depends on the mechanisms that are responsible for making the nanobubble contact angle much smaller than the macroscopic values.

\section{APPENDIX A: PROOF OF $\int_{\Sigma} \mathbf{T} \cdot \mathbf{n} d S=\mathbf{0}$}

We have

$$
\mathbf{T}=-\left(\Pi+\frac{1}{2} \epsilon_{0} \epsilon_{w} E^{2}\right) \mathbf{I}+\epsilon_{0} \epsilon_{w} \mathbf{E E} .
$$

We need to evaluate $\int_{\Sigma} \mathbf{T} \cdot \mathbf{n} d S$. Thus, let us first calculate $\int_{\Sigma}\left(\epsilon_{0} \epsilon_{w} \mathbf{E E} \cdot \mathbf{n}\right) d S$.

Applying the divergence theorem (for a volume $\Omega$ bounded by the surface $\Sigma$ ),

$$
\int_{\Sigma}\left(\epsilon_{0} \epsilon_{w} \mathbf{E E} \cdot \mathbf{n}\right) d S=\int_{\Omega} \epsilon_{0} \epsilon_{w} \nabla \cdot(\mathbf{E E}) d \Omega .
$$

One can now use the vector identity (with $\nabla \times \mathbf{E}=0$ for an electrostatic field):

$$
\nabla(\mathbf{E} \cdot \mathbf{E})=2 \mathbf{E} \times(\nabla \times \mathbf{E})+2 \mathbf{E}(\nabla \cdot \mathbf{E})=2 \mathbf{E}(\nabla \cdot \mathbf{E}) .
$$

One can also write

$$
\nabla \cdot(\mathbf{E E})=\mathbf{E}(\boldsymbol{\nabla} \cdot \mathbf{E})+\mathbf{E}(\boldsymbol{\nabla} \cdot \mathbf{E}) .
$$

Using Eq. (A3) in Eq. (A4),

$$
\nabla \cdot(\mathbf{E E})=2 \mathbf{E}(\nabla \cdot \mathbf{E})=\mathbf{E}(\boldsymbol{\nabla} \cdot \mathbf{E})+\frac{1}{2} \nabla(\mathbf{E} \cdot \mathbf{E}) .
$$

As $\nabla \Pi=\rho_{e} \mathbf{E}=\epsilon_{0} \epsilon_{w} \mathbf{E}(\nabla \cdot \mathbf{E})$ [using the Poisson equation, i.e., $\left.\rho_{e}=\epsilon_{0} \epsilon_{w}(\nabla \cdot \mathbf{E})\right]$, one can combine Eqs. (A1), (A2), and (A5) to obtain

$$
\begin{aligned}
\int_{\Sigma}\left(\epsilon_{0} \epsilon_{w} \mathbf{E E} \cdot \mathbf{n}\right) d S & =\int_{\Omega} \nabla\left(\Pi+\frac{1}{2} \epsilon_{0} \epsilon_{w} E^{2}\right) d \Omega \\
& =\int_{\Sigma}\left(\Pi+\frac{1}{2} \epsilon_{0} \epsilon_{w} E^{2}\right) \mathbf{I} \cdot \mathbf{n} d S .
\end{aligned}
$$

Using Eqs. (A1),(A6), one can say

$$
\int_{\Sigma} \mathbf{T} \cdot \mathbf{n} d S=0
$$

where $\Sigma$ refers to a closed surface located entirely within a single phase.

\section{APPENDIX B: CALCULATION OF $\zeta$ POTENTIAL FOR OTS-SILICON SUBSTRATE}

To obtain the $\zeta$ potential for a OTS-silicon substrate, one first needs to calculate the wall charge density and the $\zeta$ potential of a bare silica substrate. This is calculated by invoking the electric triple layer (ETL) -based model proposed by Wang and Revil [53] (the relevant chemistry is illustrated in Fig. 1 of Ref. [53]). For the sake of completeness we summarize the important equations of this model.

The chemical reactions that occur at the silica surface can be written as

$$
\begin{gathered}
\mathrm{SiOH}_{2}^{+} \longleftrightarrow \mathrm{SiOH}+\mathrm{H}^{+}, \text {Eqbm. Cons. } K_{a 1}, \\
\mathrm{SiOH} \longleftrightarrow \mathrm{SiO}^{-}+\mathrm{H}^{+}, \text {Eqbm. Cons. } K_{a 2}, \\
\mathrm{SiO}^{-}+M^{+} \longleftrightarrow \mathrm{SiOM} \text {,Eqbm. Cons. } K_{M}, \\
\mathrm{SiOH}_{2}^{+}+A^{-} \longleftrightarrow \mathrm{SiOH}_{2} \mathrm{~A}, \text { Eqbm. Cons. } K_{A} .
\end{gathered}
$$


Here $M^{+}$and $A^{-}$are the concentrations of the added cations and anions at the silica surface. Also note that the equilibrium constants $K_{a 1}$ and $K_{a 2}$ can be connected as

$$
p H_{\mathrm{pzc}}=\frac{1}{2}\left(\log _{10} K_{a 1}+\log _{10} K_{a 2}\right),
$$

where $p H_{\mathrm{pzc}}$ is the $\mathrm{pH}$ at the point of zero charge of surfaces, which is around 2-3 for bare silica surfaces.

From Eqs. (B1), (B2), (B3), and (B4), one can write

$$
\begin{gathered}
K_{a 1}=\frac{\sigma_{\mathrm{SiOH}}}{\sigma_{\mathrm{SiOH}_{2}^{+}}} C_{\mathrm{H}^{+}}^{b} \exp \left(-\frac{e \psi_{0}}{k_{B} T}\right), \\
K_{a 2}=\frac{\sigma_{\mathrm{SiO}^{-}}}{\sigma_{\mathrm{SiOH}}} C_{\mathrm{H}^{+}}^{b} \exp \left(-\frac{e \psi_{0}}{k_{B} T}\right), \\
K_{M}=\frac{\sigma_{\mathrm{SiOM}}}{\sigma_{\mathrm{SiO}^{-}}} \frac{1}{C_{M^{+}}^{b}} \exp \left(\frac{e \psi_{\beta}}{k_{B} T}\right), \\
K_{A}=\frac{\sigma_{\mathrm{SiOH}_{2} A}}{\sigma_{\mathrm{SiOH}_{2}^{+}}} \frac{1}{C_{A^{-}}^{b}} \exp \left(-\frac{e \psi_{\beta}}{k_{B} T}\right),
\end{gathered}
$$

where $\sigma_{\chi}$ is the surface charge density of a species $\chi, C_{H^{+}}^{b}$, $C_{M^{+}}^{b}$, and $C_{A^{-}}^{b}$ are the bulk concentrations (in M) of $H^{+}, M^{+}$, and $A^{-}$ions, and $\psi_{0}$ and $\psi_{\beta}$ are the potentials at the wall and at the Stern layer (see Ref. [53] for more details).

The continuity condition for the surface charge density gives

$$
e \Gamma_{0}=\sigma_{\mathrm{SiOH}}+\sigma_{\mathrm{SiO}^{-}}+\sigma_{\mathrm{SiOH}_{2}^{+}}+\sigma_{\mathrm{SiOM}}+\sigma_{\mathrm{SiOH}_{2} A},
$$

where $\Gamma_{0}$ is the total surface site density (typically having a value of 5-8 $\mathrm{nm}^{-2}$ for bare silica).

Based on the present ETL model, one can write the charge density at the silica surface as

$$
\sigma_{0}=\sigma_{\mathrm{SiOH}_{2}^{+}}-\sigma_{\mathrm{SiO}^{-}}-\sigma_{\mathrm{SiOM}}+\sigma_{\mathrm{SiOH}_{2} A},
$$

and the charge density at the Stern layer will be

$$
\sigma_{\beta}=\sigma_{\mathrm{SiOM}}-\sigma_{\mathrm{SiOH}_{2} A} .
$$

The global electroneutrality will yield

$$
\sigma_{0}+\sigma_{\beta}+\sigma_{d}=0,
$$

where $\sigma_{d}$ is the surface charge density of the diffuse layer.

Also the potentials at different sections of the ETL can be expressed as

$$
\psi_{0}-\psi_{\beta}=\sigma_{0} / C_{p, 1}
$$

and

$$
\psi_{\beta}-\psi_{d}=-\sigma_{d} / C_{p, 2},
$$

where $\psi_{d}$ is the potential at the outer plane of the Stern layer and $C_{p, 1}$ and $C_{p, 2}$ are the capacitances of the inner and the outer parts of the Stern layer, respectively. From the above formulation, it is clear that the complete system is governed by a set of 11 unknowns, namely, $\sigma_{\mathrm{SiOH}}, \sigma_{\mathrm{SiO}^{-}}, \sigma_{\mathrm{SiOH}_{2}^{+}}, \sigma_{\mathrm{SiOM}}$, $\sigma_{\mathrm{SiOH}_{2} A}, \sigma_{0}, \sigma_{\beta}, \sigma_{d}, \psi_{0}, \psi_{\beta}$, and $\psi_{d}$. For these 11 unknowns, we can readily identify 10 equations, namely, (B6), (B7), (B8), (B9), (B10), (B11), (B12), (B13), (B14), and (B15).

The eleventh condition comes from the expression of the diffuse layer charge density $\left(\sigma_{d}\right)$ expressed as [53]

$$
\sigma_{d}=-\sigma_{a}\left(\sinh \frac{e \psi_{d}}{2 k_{B} T}\right),
$$

where $\sigma_{a}=\sqrt{8000 \epsilon_{0} \epsilon_{w} k_{B} T N_{A}\left(C_{M+}^{b}+C_{H^{+}}^{b}\right)}$.

A OTS-silicon substrate is prepared by coating a layer of OTS on the bare silica substrate [51]. The main purpose of OTS treatment to bare silica is to convert the hydrophilic bare silica to hydrophobic OTS-silicon, which will allow the formation of surface nanobubbles. The exact reaction chemistry of OTS with bare silica is not considered here. Rather, we make a gross assumption that $\sigma_{0}$ for OTS-silicon substrate is some fraction $(f, 0<f<1)$ of bare silica (the larger the extent of OTS coating, the smaller is $f$ ) [51]. Also assuming the charge density of the coating layer as $\sigma_{\text {OTS }}$, one can now modify Eqs. (B10), (B11), and (B12) as

$$
\begin{gathered}
e \Gamma_{0}=\sigma_{\mathrm{SiOH}}+\sigma_{\mathrm{SiO}^{-}}+\sigma_{\mathrm{SiOH}_{2}^{+}}+\sigma_{\mathrm{SiOM}}+\sigma_{\mathrm{SiOH}_{2} A}+\sigma_{\mathrm{OTS}} \\
f \sigma_{0}=\sigma_{\mathrm{SiOH}_{2}^{+}}-\sigma_{\mathrm{SiO}^{-}}-\sigma_{\mathrm{SiOM}}+\sigma_{\mathrm{SiOH}_{2} A}+\sigma_{\mathrm{OTS}}, \\
\sigma_{\beta}=\sigma_{\mathrm{SiOM}}-\sigma_{\mathrm{SiOH}_{2} A}-\sigma_{\mathrm{OTS}} .
\end{gathered}
$$

Thus we will again have 11 equations [(B6), (B7), (B8), (B9), (B13), (B14), (B15), (B17), (B18), and (B19)] for 11 unknowns $\left[\sigma_{\mathrm{SiOH}}, \sigma_{\mathrm{SiO}^{-}}, \sigma_{\mathrm{SiOH}_{2}^{+}}, \sigma_{\mathrm{SiOM}}, \sigma_{\mathrm{SiOH}_{2} A}, \sigma_{\mathrm{OTS}}, \sigma_{\beta}, \sigma_{d}\right.$, $\psi_{0}, \psi_{\beta}$, and $\psi_{d}$ ], which are now iteratively solved to obtain the $\zeta$ potential $\left(\psi_{d}\right)$ for the OTS-silicon substrate.

As a final remark, we should like to mention here that for very alkaline $\mathrm{pH}$ some of the above equations may not always be sufficient to capture the electrochemistry of the silica surfaces, and one needs to account for the nontrivial reactions of the excess $\mathrm{OH}^{-}$ions with $\mathrm{SiOH}$. However, in the present calculation we neglect such an effect.
[1] M. Hampton and A. Nguyen, Miner. Eng. 22, 786 (2009).

[2] J. W. G. Tyrrell and P. Attard, Phys. Rev. Lett. 87, 176104 (2001).

[3] J. W. G. Tyrrell and P. Attard, Langmuir 18, 160 (2002).

[4] P. Attard, M. P. Moody, and J. W. G. Tyrrell, Physica A 314, 696 (2002).
[5] N. Ishida, T. Inoue, M. Miyahara, and K. Higashitani, Langmuir 16, 6377 (2000).

[6] M. Holmberg, A. Kuhle, J. Garnaes, K. A. Morch, and A. Boisen, Langmuir 19, 10510 (2003).

[7] S. Yang, S. Dammer, N. Bremond, H. J. W. Zandvliet, E. S. Kooij, and D. Lohse, Langmuir 23, 7072 (2007). 
[8] S. Yang, E. S. Kooij, B. Poelsema, D. Lohse, and H. J. W. Zandvliet, Europhys. Lett. 81, 64006 (2008).

[9] S. Yang, P. Tsai, E. S. Kooij, A. Prosperetti, H. J. W. Zandvliet, and D. Lohse, Langmuir 25, 1466 (2009).

[10] B. M. Borkent, H. Schönherr, G. Le Caer, B. Dollet, and D. Lohse, Phys. Rev. E 80, 036315 (2009).

[11] L. J. Zhang, Y. Zhang, X. H. Zhang, Z. X. Li, G. X. Shen, M. Ye, C. H. Fan, H. P. Fang, and J. Hu, Langmuir 22, 8109 (2006).

[12] A. Agrawal, J. Park, D. Y. Ryu, P. T. Hammond, T. P. Russel, and G. H. McKinley, Nano Lett. 5, 1751 (2005).

[13] X. H. Zhang, X. D. Zhang, S. T. Lou, Z. X. Zhang, J. L. Sun, and J. Hu, Langmuir 20, 3813 (2004).

[14] A. Simonsen, P. Hansen, and B. J. Klösgen, J. Colloid Interface Sci. 273, 291 (2004).

[15] M. Switkes and J. W. Ruberti, Appl. Phys. Lett. 84, 4759 (2004).

[16] X. H. Zhang, A. Quinn, and W. A. Ducker, Langmuir 24, 4756 (2008).

[17] X. H. Zhang, A. Khan, and W. A. Ducker, Phys. Rev. Lett. 98, 136101 (2007).

[18] P. Attard, Adv. Colloid Interface Sci. 104, 75 (2003).

[19] T. H. Fan and O. I. Vinogradova, Phys. Rev. E 72, 066306 (2005).

[20] N. Kameda and S. Nakabayashi, Chem. Phys. Lett. 461, 122 (2008).

[21] N. Kameda and S. Nakabayashi, Surf. Sci. 602, 1759 (2008).

[22] B. M. Borkent, S. M. Dammer, H. Schönherr, G. J. Vancso, and D. Lohse, Phys. Rev. Lett. 98, 204502 (2007).

[23] M. P. Brenner and D. Lohse, Phys. Rev. Lett. 101, 214505 (2008).

[24] S. Lou, Z. Ouyang, Y. Zhang, X. Li, J. Hu, M. Li, and F. Yang, J. Vac. Sci. Technol. B 18, 2573 (2000).

[25] S. Lou, J. Gao, X. Xiao, X. Li, G. Li, Y. Zhang, M. Li, J. Sun, X. Li, and J. Hu, Mater. Charact. 48, 211 (2002).

[26] R. Steitz, T. Gutberlet, T. Hauss, B. Klösgen, R. Krastev, S. Schemmel, A. C. Simonsen, and G. H. Findenegg, Langmuir 19, 2409 (2003).

[27] L. Zhang, Y. Zhang, X. Zhang, Z. Li, G. Shen, M. Ye, C. Fan, H. Fang, and J. Hu, Langmuir 22, 8109 (2006).

[28] X. H. Zhang, G. Li, N. Maeda, and J. Hu, Langmuir 22, 9238 (2006).

[29] F. Jin, J. F. Li, X. D. Ye, and C. Wu, J. Phys. Chem. B 111, 11745 (2007).

[30] L. Zhang, X. Zhang, Y. Zhang, J. Hu, and H. Fang, Soft Matt. 6, 4515 (2010).

[31] N. Canter, Tribol. Lubric. Tech. 66, 14 (2006).

[32] B. M. Borkent, S. de Beer, F. Mugele, and D. Lohse, Langmuir 26, 260 (2010)

[33] A. Habich, W. Ducker, D. Dunstan, and X. Zhang, J. Phys. Chem. B 114, 6962 (2010).

[34] A. Checco, T. Hofmann, E. DiMasi, C. T. Black, and B. Ocko, Nano Lett. 10, 1354 (2010).

[35] W. Ducker, Langmuir 25, 8907 (2009).

[36] S. Das, J. H. Snoeijer, and D. Lohse, Phys. Rev. E 82, 056310 (2010).

[37] J. R. T. Seddon, E. S. Kooij, B. Poelsema, H. J. W. Zandvliet, and D. Lohse, Phys. Rev. Lett. 106, 056101 (2011).

[38] S. Das, Phys. Rev. E 83, 066315 (2011).

[39] M. Hampton and A. V. Nguyen, Adv. Colloid Interface Sci. 154, 30 (2010).

[40] V. Craig, Soft Matter 7, 40 (2011).
[41] J. R. T. Seddon and D. Lohse, J. Phys. Condens. Matter 23, 133001 (2011).

[42] X. Zhang, Z. Wu, X. Zhang, G. Li, and J. Hu, Int. J. Nanosci. 4, 399 (2005).

[43] X. Zhang, N. Maeda, and V. Craig, Langmuir 22, 5025 (2006).

[44] K. Kang, I. Kang, and C. Lee, Langmuir 19, 5407(2003).

[45] K. Kang, I. Kang, and C. Lee, Langmuir 19, 6881(2003).

[46] K. Kang, I. Kang, and C. Lee, Langmuir 19, 9334 (2003).

[47] K. Kang and I. Kang, Langmuir 19, 9962 (2003).

[48] C. K. Hua, I. S. Kang, K. H. Kang, and H. A. Stone, Phys. Rev. E 81, 036314 (2010).

[49] R. J. Hunter, Zeta Potential in Colloid Science (Academic Press, London, 1981).

[50] F. Mugele and J.-C. Baret, J. Phys. Condens. Matter 17, R705 (2005).

[51] D. Stein, M. Kruithof, and C. Dekker, Phys. Rev. Lett. 93, 035901 (2004).

[52] S. Behrens and D. Grier, J. Chem. Phys. 115, 6716 (2001).

[53] M. Wang and A. Revil, J. Colloid Interface Sci. 343, 381 (2010).

[54] J. Buehrle, S. Herminghaus, and F. Mugele, Phys. Rev. Lett. 91, 086101 (2003).

[55] S. Patel and C. L. Brooks, J. Chem. Phys. 123, 164502 (2005).

[56] S. Patel, Y. Zhong, B. A. Bauer, and J. E. Davis, J. Phys. Chem. B 113, 9241 (2009).

[57] K. Leung, J. Phys. Chem. Lett. 1, 496 (2009).

[58] B. D. Bauer and S. Patel, J. Chem. Phys. 131, 084709 (2009).

[59] D. Horinek and R. R. Netz, Phys. Rev. Lett. 99, 226104 (2007).

[60] D. Horinek, A. Serr, D. J. Bonthuis, M. Boström, W. Kunz, and R. R. Netz, Langmuir 24, 1271 (2008).

[61] P. Jungwirth and B. Winter, Annu. Rev. Phys. Chem. 59, 343 (2008).

[62] F. H. J. van der Heyden, D. Stein, and C. Dekker, Phys. Rev. Lett. 95, 116104 (2005).

[63] B. Kirby and E. Hasselbrink Jr., Electrophoresis 25, 187 (2004).

[64] P. Scales, F. Grieser, T. Healy, L. White, and C. D. Y. C., Langmuir 8, 965 (1992).

[65] M. Kosmulski and E. Matijevic, Langmuir 8, 1060 (1992).

[66] V. Paruchuria, A. Nguyen, and J. Mille, Colloids Surf. A 250, 519 (2004).

[67] A. Gupta, D. Coelho, and P. Adler, J. Colloid Interface Sci. 316, 140 (2007).

[68] J. H. Weijs, J. H. Snoeijer, and D. Lohse (in preparation).

[69] X. H. Zhang, X. Zhang, J. Sun, Z. Zhang, G. Li, H. Fang, X. Xiao, X. Zeng, and J. Hu, Langmuir 23, 1778 (2007).

[70] X. H. Zhang, N. Maeda, and J. Hu, J. Phys. Chem. B 112, 13671 (2008).

[71] L. Zhang, X. Zhang, C. Fan, Y. Zhang, and J. Hu, Langmuir 25, 8860 (2009).

[72] J. R. T. Seddon, O. Bliznuyk, E. S. Kooij, B. Poelsema, H. W. Zandvliet, and D. Lohse, Langmuir 26, 9640 (2010).

[73] V. I. Kovalchuka and D. Vollhardt, Adv. Colloid Interface Sci. 114-115, 267 (2005).

[74] S. M. Dammer and D. Lohse, Phys. Rev. Lett. 96, 206101 (2006).

[75] J. H. Weijs, A. Marchand, B. Andreotti, D. Lohse, and J. H. Snoeijer, Phys. Fluids 23, 022001 (2011).

[76] A. Checco, P. Guenoun, and J. Daillant, Phys. Rev. Lett. 91, 186101 (2003). 